\title{
Global Optimization Algorithm Based on One-Dimensional Chaotic Maps and Gradient Descent Technique
}

\section{Tsankova, S. Lekova}

Key Words: Global optimum; multimodal function; chaos; gradient descentmethod.

\begin{abstract}
A hybrid algorithm for searching the global minimum of a multimodal function is proposed in the paper. It is a two stages search technique, the first stage is the twice carrier wave based chaotic optimization algorithm (COA) for global searching, and the second stage is the gradient descent algorithm (GDA) for accurate local searching. The chaotic dynamics is realized through one-dimensional map in three variants: logistic, cubic and sine map. Three testing functions are used. A hundred simulations (each starting from different initial point generated randomly) were carried out for each of the test functions using two optimization algorithms: the proposed hybrid algorithm and the GDA working alone. The success and accuracy of locating the extremum, as well as the convergence of the algorithms using the three different chaotic maps were discussed.
\end{abstract}

\section{Introduction}

Multimodality is a phenomenon, which occurs very often in the engineering, economic and other optimization tasks using complex high-order objective (cost) functions. Many algorithms have been developed to locate the global optimum [1], and the convergence of some of them has been mathematically proved. But there is not an effective computational algorithm, which could guarantee finding the global optimum in rational number of iterations.

Chaotic sequences have been adopted instead of random sequences to provide the search diversity in an optimization procedure, named chaos optimization algorithm (COA) [2,3]. Due to the non-repetition of the chaos, the properties as ergodicity and irregularity of chaos, the COA can carry out overall searches at higher speeds than stochastic ergodic searches that depend on the probabilities $[4,5,6,7,8]$. The COA has the features of easy implementation, short execution time and robust mechanisms of escaping from the local optimum $[4,5]$. In order to enhance the performance of the optimization search algorithms, some researchers have combined the COA with meta-heuristics algorithms, such as chaotic genetic algorithms [9], chaotic ant swarm optimization [10], chaotic particle swarm optimization $[11,12]$, chaotic harmony search algorithm $[13,4]$, chaotic firefly algorithm [14], etc.

The purpose of the paper is to propose a new hybrid algorithm for a global optimization of multimodal objective functions. The proposed hybrid algorithm is a two stages search technique, the first stage is the COA with twice carrier wave $[4,5]$ chaos search for global searching, and the second stage is the classical gradient descent algorithm (GDA) for accurate local searching. The COA is conducted until it has converged to a close neighborhood or it has reached its maximum iteration times, then the GDA is conducted for fast and accurate solution. Three different types of chaotic map (logistic, cubic and sinusoidal) are used to produce chaotic sequences for the COA. The proposed hybrid optimization algorithm is tested in MATLAB environment.

\section{Chaotic Optimization Approach}

\subsection{Chaotic Maps}

Chaotic maps are usually used for modeling chaos. A chaotic map is a dynamical discrete-time continuous value function, which defines the relationship between the current and following state of a chaotic system [14]. One dimensional noninvertible maps have capability to generate chaotic motion [4]. In this study, three well-known onedimensional chaotic maps are considered.

Logistic map is one of the most frequently used chaotic maps in the optimization procedures [11]. This map has been introduced by Robert May in 1976 [15] when he has investigated the fluctuations of insect populations. The map is defined by the following equation:

$$
\text { (1) } x(k+1)=\varphi x(k)(1-x(k)), 0<\varphi \leq 4, x(k) \in(0,1) \text {. }
$$

Motivated by a problem in genetics May [16] has given another example of chaotic map - cubic map $[17,18]$. It is used in generating chaotic sequences in various applications like cryptography. The description of the map is [4]

(2) $x(k+1)=\rho x(k)\left(1-x(k)^{2}\right), \rho=2.59, x(k) \in(0,1)$.

Sine map introduced by Peitgen et al. [19] is formally defined by the following equation:

$$
\text { (3) } x(k+1)=\sin (\pi x(k)), x(k) \in(0,1) \text {. }
$$

Typical behaviors of one chaos variables $\left(x_{1}\right)$ based on different chaotic maps with 100 iterations are shown in figure 1. Here the initial point of the chaos variable is: $x_{1}=0.1$. Figure $1(a)$ is the result of logistic map $(\varphi=4)$, figure 1(b) - cubic map, and figure 1(c) - sine map. All the three maps generate chaotic sequences in the interval $(0,1)$. The distribution or ergodic property of 

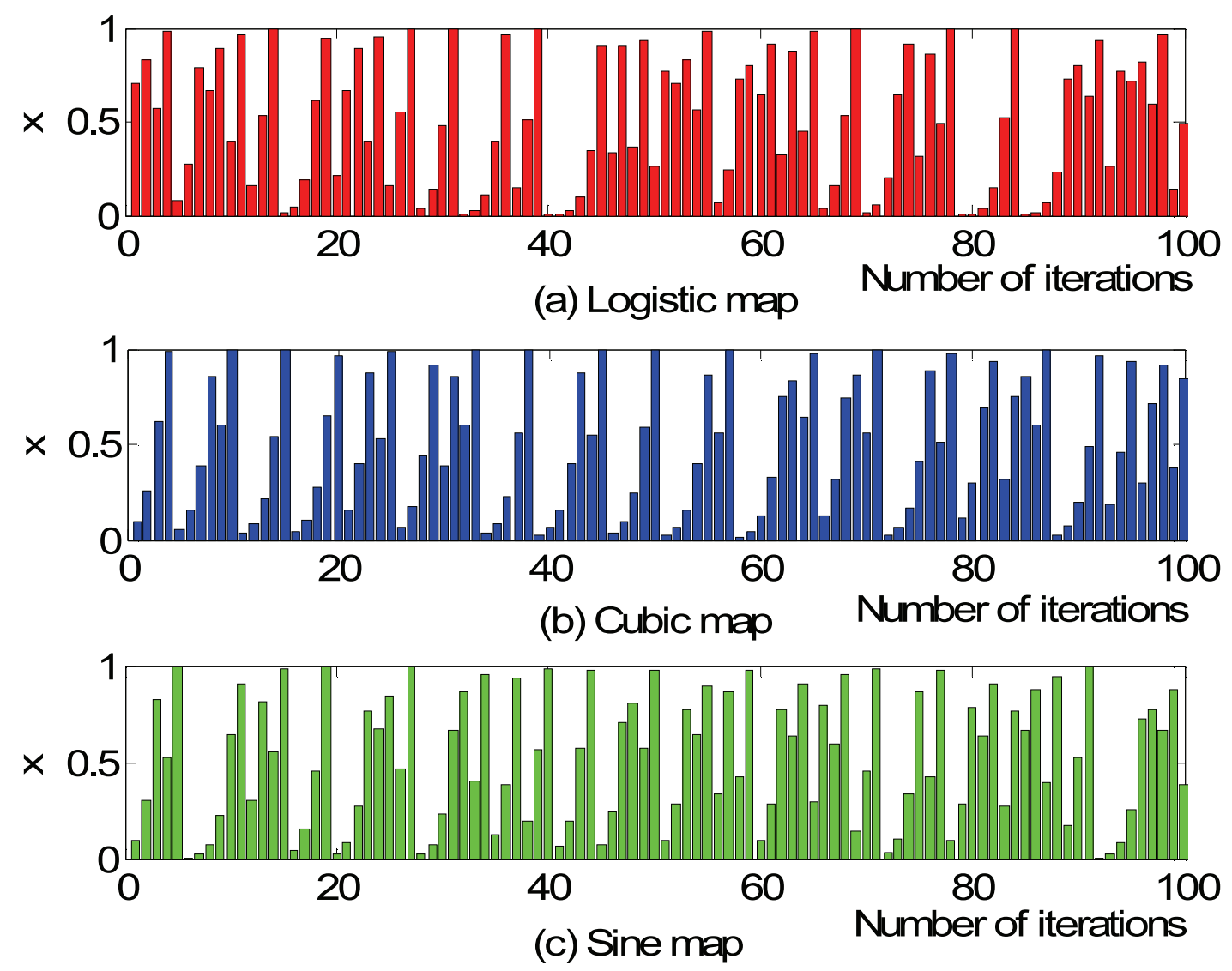

Figure 1. Bar plots of one chaos variable

different chaotic maps are different [4]. Therefore, the search patterns of different chaotic maps differ with each others in view of convergence rate, algorithm speed and accuracy $[4,11,6]$.

\subsection{Chaotic Optimization Algorithm}

Consider the problem of locating the global optimum (minimum) $\mathbf{x}=\mathbf{x}^{*}$ of a nonlinear multimodal function $Q(\mathbf{x})$, where $\mathbf{x}=\left(x_{1}, x_{2}, \ldots, x_{n}\right)^{\mathrm{T}}$, with boundary constraints $x_{i} \in\left[a_{i}, b_{i}\right]$, for $i=1,2, \ldots n$. Here, the twice carrier wave COA approach is presented as it is proposed in [4,5]. The first is the raw searching in different chaotic trace, while the second is elaborate searching by continually reducing the searching space of variable optimized and enhancing the searching precision.

\section{Chaos search by using the first carrier wave:}

Step 1. Initialize the maximum iteration times $S_{1}$ in the first carrier wave chaos search, random initial value of chaotic maps $0<\gamma_{i}(0)<1$.

Step 2. Set iteration times $k=0$, optimum $Q^{*}=10^{9}$.

Step 3. Map chaotic maps $\gamma_{i}(k)$ onto the variance range of the optimization variables by the following equation:

(4) $x_{i}(k)=a_{i}+\gamma_{i}(k)\left(b_{i}-a_{i}\right)$, where $\mathbf{x}(k)=\left(x_{1}(k), x_{2}(k), \ldots, x_{n}(k)\right)^{\mathrm{T}}$.

Step 4. Compute the objective function value for the decision variable and update the searching results. If
$Q(\mathbf{x}(k))<Q^{*}$, then $\mathbf{x}^{*}=\mathbf{x}(k)$ and the global optimum $Q^{*}=Q(\mathbf{x}(k))$.

Step 5. Generate next values of the chaotic map by a chaotic map function (M) as in (1) - (3)

(5) $\gamma_{i}(k+1)=M\left(\gamma_{i}(k)\right)$.

Step 6. If $k \geq S_{1}$ stop the first carrier wave search process; otherwise $k \leftarrow k+1$, go to Step 3 .

Chaos search by using the second carrier wave

Step 1. Initialize the maximum iteration times $S_{2}$ in the second carrier wave chaos search, set iteration times $k^{\prime}=$ 0 , random initial value of chaotic maps $0<\gamma_{i}\left(k^{\prime}\right)<1$.

Step 2. Compute the second carrier wave by the following equation:

(6) $x_{i}\left(k^{\prime}\right)=x_{i}^{*}+\lambda_{i}\left(\gamma_{i}\left(k^{\prime}\right)-0.5\right)$.

Step 3. Compute the objective function value for the decision variable and update the searching results. If $Q\left(\mathbf{x}\left(k^{\prime}\right)\right)<Q^{*}$, then $\mathbf{x}^{*}=\mathbf{x}\left(k^{\prime}\right)$ and the global optimum $Q^{*}=Q(\mathbf{x}(k))$.

Step 4. Generate next values of the chaotic map by a chaotic map function as in (1) - (3)

(7) $\gamma_{i}\left(k^{\prime}+1\right)=M\left(\gamma_{i}\left(k^{\prime}\right)\right)$.

Step 5. If $k^{\prime} \geq S_{2}$, stop the second carrier wave search process; otherwise $\lambda_{i} \leftarrow t \lambda_{i}, k^{\prime} \leftarrow k^{\prime}+1$, go to Step 2 .

The $\lambda$ is a very important parameter that adjusts a 
small ergodic range around $\mathbf{x}^{*}$, and $k>1$. The appropriate value of $\lambda$ is determined heuristically; initial value of this parameter is usually set to $0.01\left(b_{i}-a_{i}\right)[4,5]$.

\section{Hybrid Chaotic Optimization Algorithm with Gradient Descent Algorithm}

The motion step of chaotic map between two successive iterations is always big, thus, even if COA has reached the neighbourhood of the global optimum, it needs to spend much computational effort to approach the optimum by searching numerous points. In order to improve the accurate local search of COA, a classical gradient descent algorithm (GDA) is employed here. The flowchart of hybrid COA with GDA is illustrated in figure 2. The proposed hybrid algorithm is a two stages search technique, the first stage is the twice carrier wave COA for global searching, and the second stage is the GDA for accurate searching. The condition of switch from COA to GDA is: the second carrier wave chaos search is completed $\left(k^{\prime} \geq S_{2}\right)$. The optimum $Q^{*}$ (that is, $\mathbf{x}^{*}$ after COA search) is the starting point of GDA.

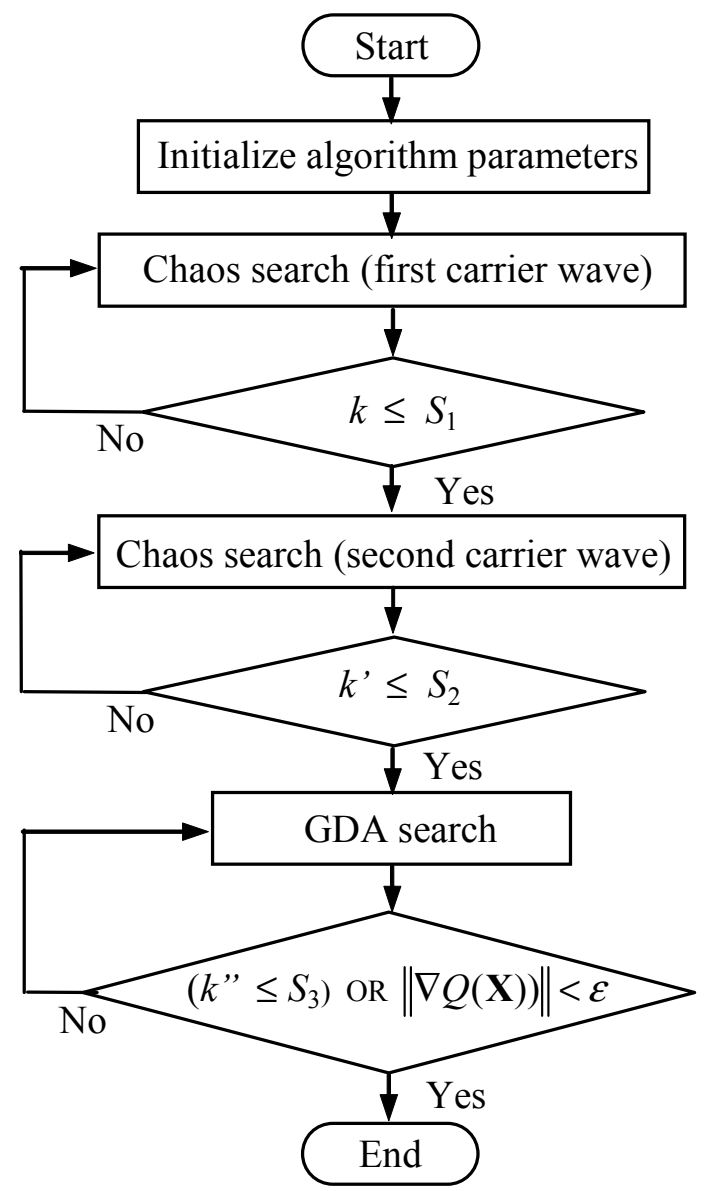

Figure 2. Flowchart of the proposed hybrid COA with GDA

\subsection{Gradient Descent Algorithm}

This section describes the GDA after COA search as follows. Let the real-valued function $Q(\mathbf{x})$ be differentiable in respect to $\mathbf{x}$. The derivatives vanish at the extrema of the function, i.e.

(8) $\frac{\partial Q(\mathbf{x})}{\partial x_{i}}=0, \quad i=1,2, \ldots, n$.

The solutions depend on the choice of the initial guess and often correspond to a local optimum. The fastest decrease of $Q(\mathbf{x})$ can be obtained in the direction of the negative gradient of $Q(\mathbf{x})$, i.e.

(9) $\frac{d x_{i}}{d t}=-\frac{\partial Q(\mathbf{x})}{\partial x_{i}}, \quad i=1,2, \ldots, n$.

\section{Gradient descent algorithm}

Step 1. Initialize GDA parameters: maximum iteration times $S_{3} ; h_{i}(i=1,2, \ldots, n)$ - small positive constants (the size of the steps along the gradient direction); $\varepsilon$ - a small positive number for the stop criterion.

Step 2. Set iteration times $k^{\prime \prime}=0$; objective function $Q(0)=Q^{*}$ and $\mathbf{x}(0)=\mathbf{x}^{*}$.

Step 3. Calculate the optimization variables by the gradient descent technique (presented in discrete times)

$$
\begin{aligned}
& x_{i}\left(k^{\prime \prime}+1\right)=x_{i}\left(k^{\prime \prime}\right)-\frac{h_{i}\left(k^{\prime \prime}\right)}{\left\|\nabla Q\left(\mathbf{x}\left(k^{\prime \prime}\right)\right)\right\|} \cdot \frac{\partial Q(\mathbf{x})}{\partial x_{i}}\left(k^{\prime \prime}\right), \\
& i=1,2, \ldots, n
\end{aligned}
$$

where

$$
\nabla Q(\mathbf{x})=\left[\frac{\partial Q(\mathbf{x})}{\partial x_{1}}, \frac{\partial Q(\mathbf{x})}{\partial x_{2}}, \ldots \frac{\partial Q(\mathbf{x})}{\partial x_{n}}\right]^{\mathrm{T}}
$$

and

$$
\|\nabla Q(\mathbf{x})\|=\sqrt{\sum_{i=1}^{n}\left(\frac{\partial Q(\mathbf{x})}{\partial x_{i}}\right)^{2}} .
$$

Step 4. Compute the objective function value for the decision variable and update the searching results. If $Q\left(\mathbf{x}\left(k^{\prime \prime}\right)\right)<Q^{*}$, then $\mathbf{x}^{*}=\mathbf{x}\left(k^{\prime \prime}\right)$ and the global optimum $Q^{*}=Q\left(\mathbf{x}\left(k^{\prime \prime}\right)\right)$.

Step 5. If $k^{\prime \prime} \geq S_{3}$, or ||$\nabla Q(\mathbf{x}(k))|| \leq \varepsilon$, then stop the GDA and print $Q^{*}$ and $\mathbf{x}^{*}$; otherwise $k^{\prime \prime} \leftarrow k^{\prime \prime}+1$ and go to Step 3 .

\section{Simulation Results and Discussions}

To illustrate the performance of the proposed hybrid chaotic optimization algorithm with gradient descent technique three global optimization tasks were simulated in MATLAB environment. The objective functions used for the test examples were $[2,4]$ :

$$
\begin{aligned}
& \text { (11) } Q_{1}(\mathbf{x})=\left(x_{1}{ }^{2}+x_{2}-11\right)^{2}+\left(x_{1}+x_{2}{ }^{2}-7\right)^{2}+\left(x_{1}-3\right)^{2}+\left(x_{2}-2\right)^{2} \text {, } \\
& -5<x_{1}, x_{2}<5
\end{aligned}
$$


(12) $Q_{2}(\mathbf{x})=\sum_{i=1}^{3}\left(x_{i}^{2}-10 \cos \left(2 \pi x_{i}\right)+10\right),-5<x_{i}<5$, $i=1,2,3$.

(13) $Q_{3}(\mathbf{x})=\frac{1}{4000} \sum_{i=1}^{30} x_{i}{ }^{2}-\prod_{i=1}^{30} \cos \left(\frac{x_{i}}{\sqrt{i}}\right)+1,-5<x_{i}<5$, $i=1,2, \ldots, 30$.

Function $Q_{1}$ is illustrated in figure $3(a)$, it has four

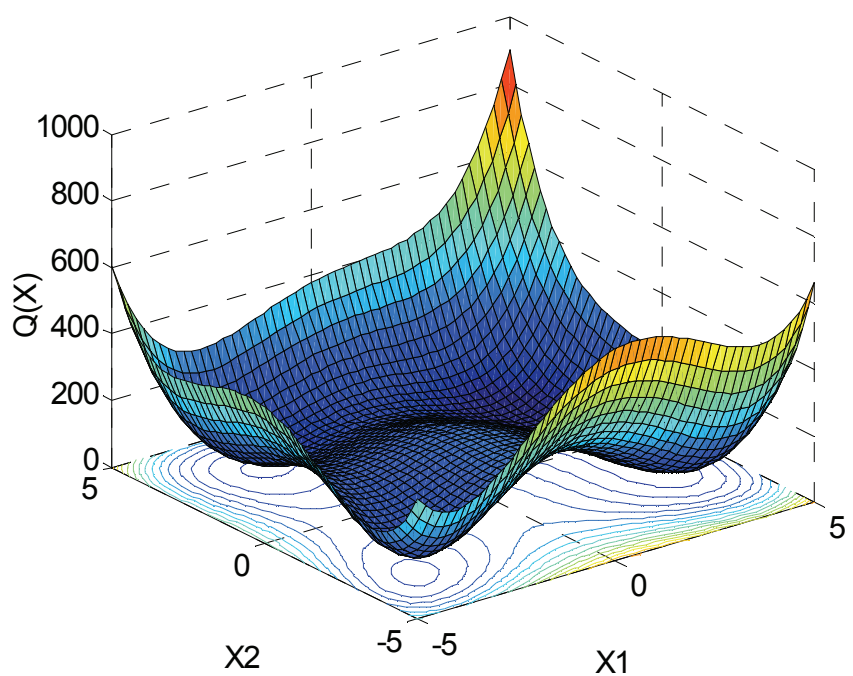

(a) $Q_{1}$
The derivatives of the objective functions (11), (12) and (13) used in (10) are calculated as follows:

$$
\frac{\partial Q_{1}(\mathbf{x})}{\partial x_{1}}=4 x_{1}^{3}+2 x_{2}^{2}+4 x_{1} x_{2}-40 x_{1}-20 ;
$$

(14) $\frac{\partial Q_{1}(\mathbf{x})}{\partial x_{2}}=4 x_{2}^{3}+2 x_{1}^{2}+4 x_{1} x_{2}-24 x_{2}-26$;

(15) $\frac{\partial Q_{2}(\mathbf{x})}{\partial x_{i}}=2 x_{i}+20 \pi \sin \left(2 \pi x_{i}\right), \quad i=1,2,3$;

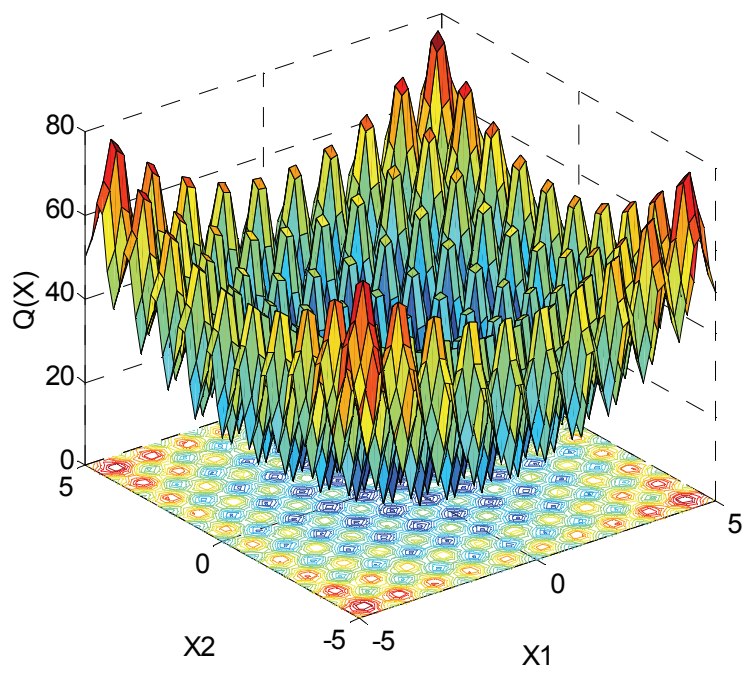

(b) $Q_{2}$

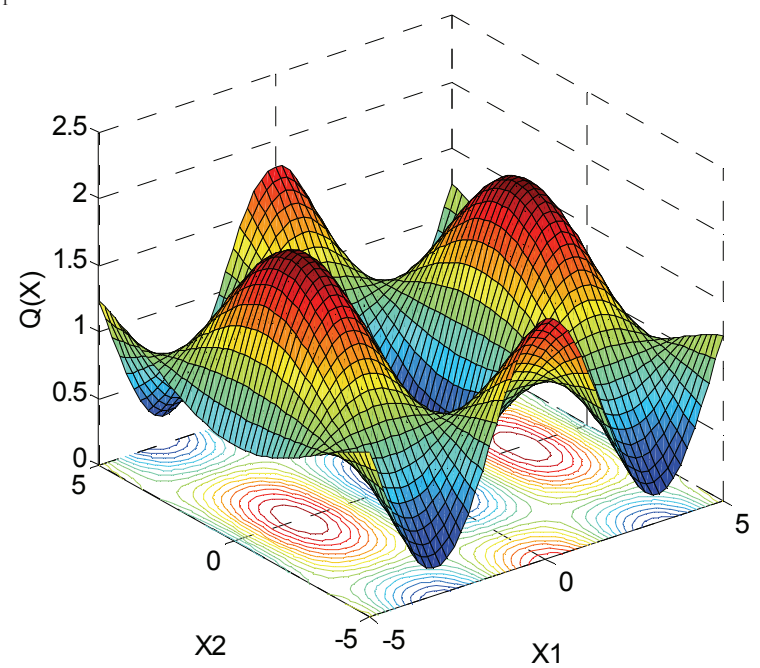

(c) $Q_{3}$

Figure 3. $Q_{1}, Q_{2}$ and $Q_{3}$ functions in two-dimension space

local minima and one global minimum $\mathbf{x}^{*}=(3,2)$, and optimal objective function value $Q_{1}{ }^{*}=0$. Function $Q_{2}$ is the Rastrigin's function as illustrated in figure $3(b)$, which has many local minima and one global minimum $\mathbf{x}^{*}=(0,0,0)$, and $Q_{2}{ }^{*}=0$. Function $Q_{3}$ is the Griewank's function with 30 variables as illustrated in figure 3(c), which has several thousand local minima and one global minimum $\mathbf{x}^{*}=(0,0, \ldots, 0)$, and $Q_{3}{ }^{*}=0$.

$$
\text { (16) } \begin{aligned}
& \frac{\partial Q_{3}(\mathbf{x})}{\partial x_{i}}=\frac{1}{2000} x_{i}+\frac{1}{\sqrt{i}} \sin \left(\frac{x_{i}}{\sqrt{i}}\right) \prod_{\substack{j=1 \\
j \neq i}}^{30} \cos \left(x_{j}\right), \\
& i=1,2, \ldots, 30 .
\end{aligned}
$$

A hundred simulations (each starting from different initial point generated randomly) were carried out for each of the test functions using two optimization algorithms: (1) the proposed hybrid algorithm combining COA and GDA, 


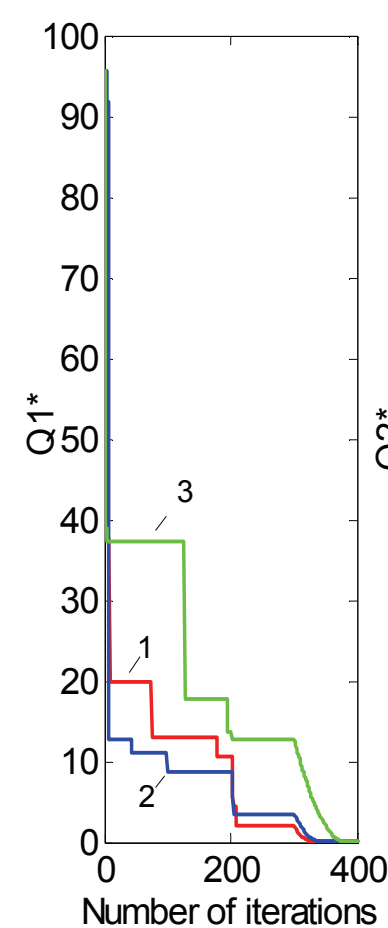

(a)

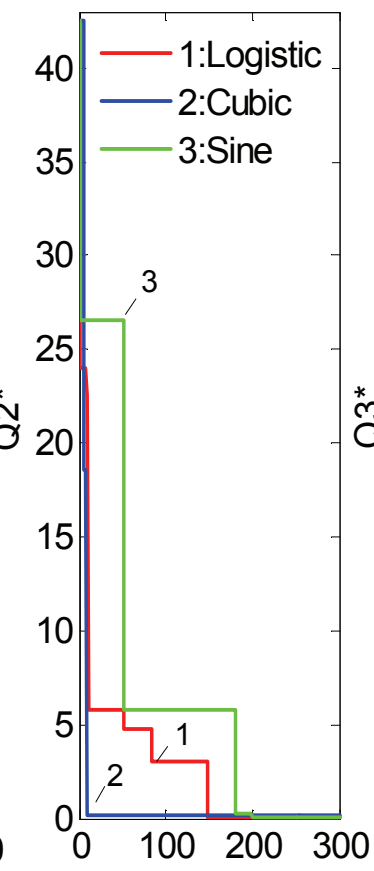

Number of iterations

(b)

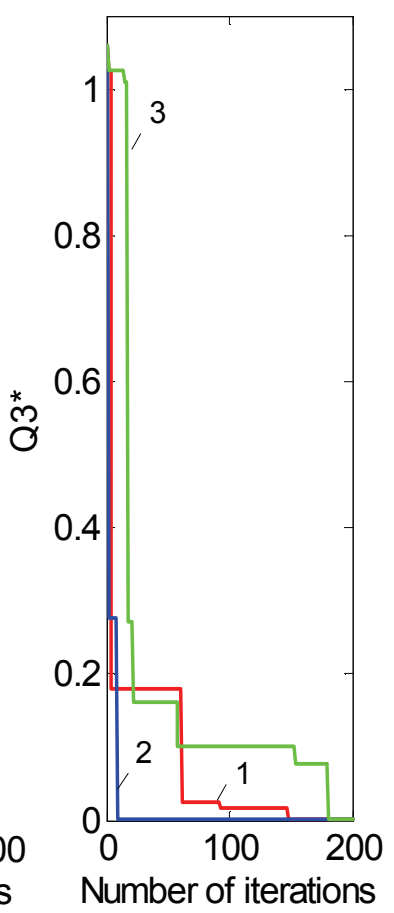

(c)

Figure 4. The convergence of the objective function's optimal value $Q^{*}$ through iterations

initial point generated randomly) were carried out for each of the test functions using two optimization algorithms: (1) the proposed hybrid algorithm combining COA and GDA, and (2) GDA. The second algorithm serves as a benchmark for determining the benefits of the proposed hybrid algorithm. COA alternatively used the three one-dimensional chaotic maps (logistic, cubic and sine map). For the simulations the following values of parameters were selected: $S_{1}=S_{2}=800, S_{3}=5000, h_{i}=0.005\left(h_{i}=0.005\right.$ for determining $\left.Q_{1}{ }^{*}\right)$. Appropriate values of these parameters are usually chosen by trial with multiple runs until finding the ones which yield appropriate result. The results obtained are shown in table 1. The accuracy of localization of the extrema for the three sets is in the range from $10^{-7}$ to $10^{-4}$. The success rate is almost $100 \%$ except for the variant using the sine map in test function $Q_{2}$. It is known, GDA working alone gets stuck in a local minimum - this happened in more than $62 \%$ of the cases under consider- ation (the test functions and the initial conditions).

Figure 4 shows the convergence of the objective function's optimal value $Q^{*}$ through iterations (for the three testing functions) using the proposed variants of the hybrid optimization algorithm. In that hybrid algorithm COA provides finding a global minimum with a reasonable number of iterations and GDA provides its localization with sufficient accuracy. COA with cubic map demonstrates a little better convergence of the optimal value $Q^{*}$ through iterations than COA with the others maps, but probably the result depends on the type of objective function, so that more experiments with more objective functions are needed.

\section{Conclusion}

A hybrid approach for localization of the global minimum of a multimodal objective function is presented. It is

Table 1. The success ratio in searching the global minimum

\begin{tabular}{|c|c|c|c|c|c|c|c|c|}
\hline & \multicolumn{2}{|c|}{ GDA } & \multicolumn{2}{c|}{$\begin{array}{c}\text { COA(Logistic) }+ \\
\text { GDA }\end{array}$} & \multicolumn{2}{c|}{ COA(Cubic) + GDA } & \multicolumn{2}{c|}{ COA(Sine) + GDA } \\
\cline { 2 - 9 } & $Q^{*}$ & $\begin{array}{c}\text { Success } \\
\mathbf{\%}\end{array}$ & $Q^{*}$ & $\begin{array}{c}\text { Success, } \\
\%\end{array}$ & $Q^{*}$ & $\begin{array}{c}\text { Success, } \\
\%\end{array}$ & $Q^{*}$ & $\begin{array}{c}\text { Success, } \\
\%\end{array}$ \\
\hline$Q_{1}(\mathbf{x})$ & $2.25 \times 10^{-7}$ & 56 & $2.07 \times 10^{-7}$ & 100 & $3.22 \times 10^{-7}$ & 100 & $3.50 \times 10^{-7}$ & 100 \\
\hline$Q_{2}(\mathbf{x})$ & $4.64 \times 10^{-4}$ & 9 & $4.37 \times 10^{-4}$ & 100 & $4.18 \times 10^{-4}$ & 100 & $4.46 \times 10^{-4}$ & 96 \\
\hline$Q_{3}(\mathbf{x})$ & $8.04 \times 10^{-7}$ & 48 & $1.55 \times 10^{-6}$ & 100 & $1.80 \times 10^{-6}$ & 100 & $1.37 \times 10^{-6}$ & 100 \\
\hline Averaged success: & 37.7 & & 100 & & 100 & & 98.7 \\
\hline
\end{tabular}


a combination of the COA for global searching and the classical GDA for accurate local searching. Logistic map was used to provide chaotic dynamics. Hundreds simulations (each starting from different initial point generated randomly) were carried out for three benchmarks test functions using two optimization algorithms: the proposed hybrid algorithm and GDA working alone. It is observed that considerable performance improvement is possible by the proposed hybrid algorithm. The hybrid algorithm has better performance than GDA: $99.6 \%$ success in locating the global optimum vs. $37.7 \%$. All the results are averaged over the three test functions and the three different chaotic maps. The performance of the sine map is a little worse than the logistic and cubic maps: $98.7 \%$ vs. $100 \%$ success in locating the global optimum.

The future work will include further experiments with new objective functions and other chaotic maps, studies about the condition of switch from COA to GDA, discussions on the necessity, advantages and disadvantages of the parallel search algorithms.

\section{Acknowledgements}

The paper presents research and development, supported by Scientific Fund of Internal Competition of the University of Food Technologies - Plovdiv under the Research Project No. 7/16-H and the Science \& Research Programme of the University of Chemical Technology and Metallurgy - Sofia (Project 11588/2016).

\section{References}

1. Dixon, L. C. W., G. P. Szego (Eds.). Towards Global Optimization. North-Holland Publishing, Co., 1975.

2. Yang, D. X., G. Li, G. D. Cheng. On the Efficiency of Chaos Optimization Algorithms for Global Optimization. - Chaos, Solitons and Fractals, 34, 2007, No. 4, 1366-1375.

3. Yang, D., Z. Liu, J. Zhou. Chaos Optimization Algorithms Based on Chaotic Maps with Different Probability Distribution and Search Speed for Global Optimization. - Communications in Nonlinear Science and Numerical Simulation, 19, 2014, No. 4, 1229-1246.

4. Yuan, X., J. Zhao, Y. Yang, Y. Wang. Hybrid Parallel Chaos Optimization Algorithm with Harmony Search Algorithm. - Applied Soft Computing, 17, 2014, 12-22.

5. Yuan, X., T. Zhang, Y. Xiang, X. Dai. Parallel Chaos Optimization Algorithm with Migration and Merging Operation. - Applied Soft Computing, 35, 2015, 591-604.

6. Tavazoei, M. S., M. Haeri. Comparison of Different One-dimensional Maps as Chaotic Search Pattern in Chaos Optimization Algorithms. - Applied Mathematics and Computation, 187, 2007, No.2, 1076-1085.

7. Yang, D. X., Z. J. Liu, J. L. Zhou. Chaos Optimization Algorithms Based on Chaotic Maps with Different Probability Distribution and Search Speed for Global Optimization. - Communications in Nonlinear Science and Numerical Simulation, 19, 2014, No. 4, 1229-1246.

8. Naanaa, A. Fast Chaotic Optimization Algorithm Based on Spatiotemporal Maps for Global Optimization. - Applied Mathematics and Computation, 269, 2015, 402-411.

9. Ma, Z. Chaotic Populations in Genetic Algorithms. - Applied Soft Computing, 12, 2012, No. 8, 2409-2424.

10. Wan, M., C. Wang, L. Li, Y. Yang. Chaotic ant Swarm
Approach for Data Clustering. - Applied Soft Computing, 12, 2012, No. 8, 2387-2393.

11. Alatas, B., E. Akin, A. B. Ozer. Chaos Embedded Particle Swarm Optimization Algorithms. - Chaos, Solitons and Fractals, 40, 2009, No. 4, 1715-1734.

12. Tatsumi, K., T. Ibuki, T. Tanino. Particle Swarm Optimization with Stochastic Selection of Perturbation-based Chaotic Updating System. - Applied Mathematics and Computation, 269, 2015, 904-929.

13. Alatas, B. Chaotic Harmony Search Algorithms. - Applied Mathematics and Computation, 216, 2010, No. 9, 2687-2699.

14. Baykasoglu, A., F. B. Ozsoydan. Adaptive Firefly Algorithm with Chaos for Mechanical Design Optimization Problems. Applied Soft Computing, 36, 2015, 152-164.

15. May, R. M. Simple Mathematical Models with Very Complicated Dynamics. - Nature, 261, 1976, 459-467.

16. May, R. M. Biological Populations with Non Overlapping Generations: Stable Points, a Stable Cycles and Chaos. - Science, 86, 1974, 645-647.

17. Rogers, T. D., D. C. Whitley, Chaos in the Cubic Mapping. Mathematical Modeling, 4, 1983, USA, 9-25.

18. De Oliveira, J. A., E. R. Papesso, E. D. Leonel. Relaxation to Fixed Points in the Logistic and Cubic Maps: Analytical and Numerical Investigation. - Entropy, 15, 2013, 4310-4318.

19. Peitgen, H., H. Jurgens, D. Saupe. Chaos and Fractals. Berlin, Springer-Verlag, 1992.929.

\section{Manuscript received on 02.11.2016}

Diana Tsankova has an MSc degree in control systems engineering (automation of production) from the University of Food Technologies, Plovdiv. In 2002 she received her PhD degree in artificial intelligence from the Technical University of Sofia-Plovdiv Branch. She worked as an Associated Professor at the same university till 2013. Currently, she is a full time Professor at the University of Food Technologies. Her research interests cover various scientific areas, such as: AI, biologically inspired robotics, and control theory (nonlinear, optimal and adaptive systems). Recently she has developed an interest in the field of quality control of foods based on spectral analyses and pattern recognition.

Contacts:

Department of Automation, Information and Control Systems University of Food Technologies (UFT) 26 Maritsa Blvd., 4000 Plovdiv, Bulgaria e-mail:dtsankova@yahoo.com

Svetla Lekova, PhD, is currently working as an Assistant Professor in the Department of Automatics at University of Chemical Technology and Metallurgy. She received her MSc and PhD degrees in Automation of Industrial Processes from the University of Chemical Technology and Metallurgy, Sofia, Bulgaria. Her main research interests include: applied statistics; identification and system parameter estimation; simulation and modeling of processes and systems; linear and nonlinear control systems and dynamical system approximation.

Contacts:

Department of Automation of Production University of Chemical Technology and Metallurgy 8 Kliment Ohridski Blvd., 1756 Sofia, Bulgaria e-mail:sv_lekova@uctm.edu 